\title{
ELECTRON CLOUD AND PRESSURE RISE SIMULATIONS FOR RHIC*
}

\author{
U. Iriso-Ariz ${ }^{\dagger}$, M. Blaskiewicz, A. Drees, W. Fischer, S. Peggs, D. Trbojevic. \\ BNL, Upton, NY 11973, USA
}

\section{Abstract}

Beam induced electron multipacting may be among the main reasons for the vacuum pressure rise when circulating high intensity ion and proton beams in RHIC. Latest simulation results are benchmarked with recent experimental observations for RHIC, and compared to other general computer codes. The influence of the electron multipacting to the vacuum properties is also discussed.

\section{INTRODUCTION}

Pressure $(P)$ rises were observed as bunch currents were increased during both gold ( $\mathrm{Au}$ ) and proton (p) operations at the Relativistic Heavy Ion Collider (RHIC). Preliminary indications from the RHIC 2001 run [1] suggest that an electron cloud $(E C)$ may be the main cause of $P$ rises that limit the beam intensity at RHIC. Ion desorption and beam loss may also be partially responsible. In the following, we benchmark the latest experimental observations with one of the existing computing codes, CSEC( [2]). Preliminary results obtained from $E C L O U D$ [3] are also discussed. Following the results obtained by $C S E C$ and the experimental data, we give a reliable ranges for the main wall surface parameters contributing to the effect, which are necessary to determine the behaviour of the secondary electron emission, $\delta$ (Sec. 2). Observations have been made with the same fill pattern (106ns bunch spacing) and during the same process (injection, where bunches are typically $15 \mathrm{~ns}$ head-to-tail long) as in the 2001 run. Due to the bake out carried out during the RHIC shutdown in 2002, the 55 bunch fills (with 216 ns bunch spacing) did not show $E C$ effects. Due to the high $P$ rise observed in some cases, the number of injected bunches injected did not reach the target of 110 . We also compare the simulation results with data when a solenoidal field is applied to suppress the effect. The experimental results are based on the electron detector in [4]. The direct output provided by this detector is a voltage, which can be converted to a current into the wall $\left(I_{\text {wall }}\right)$ with a large uncertainty (factors $\approx 3$ are possible [4]). Despite this large error, it has been decided to show the experimental observations in terms of $I_{\text {wall }}$, expressed in $\left(\frac{\mu \mathrm{A}}{\mathrm{cm}^{2}}\right)$. Finally, and based in the experimental data, we discuss the influence of $I_{\text {wall }}$ to the $P$ for when the latter reaches a final steady state.

\footnotetext{
* Work supported by US DOE under contract DE-ACO2-98CH10886

† ubaldo@bul.gov
}

\section{PARAMETRIZATION OF THE SECONDARY EMISSION YIELD}

Following the notation in [5], for a normally incident electron with kinetic energy $E, \delta(E)$ is the total number of electrons leaving the surface due to all processes. The parametrization of $\delta(E)$ is one of the main concerns while simulating the $E C$ effect, and there are still a lot of investigations currently being carried out [7]. CSEC uses the following [2]:

$$
\begin{aligned}
\delta(E) & =\left(R-R_{\text {inf }}\right) \exp ^{-E / E_{\tau}}+R_{\text {inf }} \\
& +S E Y_{\max } \frac{s\left(E / E_{\max }\right)}{s-1+\left(E / E_{\max }\right)^{s}},
\end{aligned}
$$

where $E_{r}, s$, and $R_{i n f}$ are fitting parameters that have been fixed from [2] and [5] to $60 \mathrm{eV}, 1.813$ and 0.2 , respectively. $E_{\max }$ corresponds to the energy at which $\delta$ has its maximum value $\left(\delta_{\max }\right)$. For stainless steel (RHIC), $E_{\max }=300 \mathrm{eV}$ [6]. The contributions to $\delta(E)$ can be divided into the reflected electrons (terms proportional to $R$ and $R_{\text {inf }}$ ) and the 'true' secondaries (terms proportional to $\left.S E Y_{\max }\right)$. $R$ stands to the electron backscattering probability at low $E$, whereas $R_{i n f}$ refers for the same probability for high $E$ electrons. The angular distribution of the secondary electrons is also taken into account and can be seen in [2]. Figure 1 illustrates the global behaviour of $\delta$ and the individua contribution of the reflected electrons and the true secondaries, respectively.

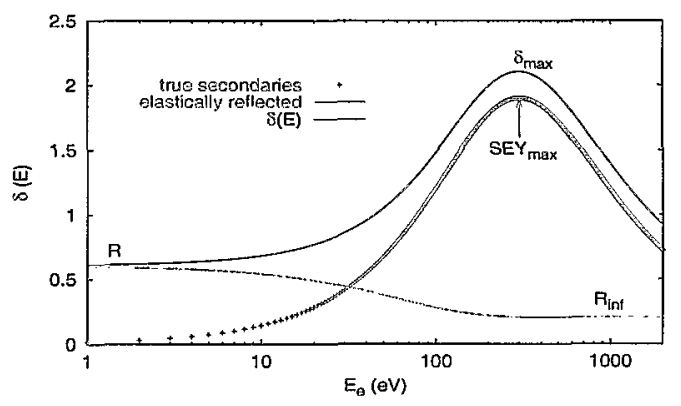

Figure 1: Separation between the processes influencing $\delta$ for $\mathrm{R}=0.6$ and $S E Y_{\max }=1.9$.

$E C$ is very sensitive to the $S E Y_{\max }$ and $R$. By comparing $C S E C$ results with experimental observations, we give a reliable range for these $S E Y_{\max }$ and $R$ referring to the RHIC beam pipe wall. On the other hand, the freedom to change these specific parameters in the input file using the $E C L O U D$ code is reduced only to the value $\delta_{\max }$ (Fig. 1). Note that $\delta_{\max }$ is not the same as the $S E Y_{\max }$ from $C S E C$. The way in which the reflected electrons are 
evaluated in ECLOUD is fixed. The parametrization used there has been updated following laboratory measurements. The latest version, which is the one used here, can be seen in [7].

\section{SIMULATIONS COMPARED WITH EXPERIMENTAL DATA}

Clear $E C$ cases occurred during the RHIC FY2003 polarized proton $(p p)$ run, where it was technically feasible to reach higher bunch intensities $\left(I_{p b}\right)$ than during the $d A u$ run. These cases occurred at the long straight section labeled 'bo2', where the vacuum chamber has cylindrical symmetry. The main machine characteristics, together with the beam parameters for fill \#3460 can be seen at Table 1 . Figure 2 shows a snapshot of the $E C$ signal collected in the $E D$ and the bunch intensity $\left(I_{p b}\right)$ for fill \#3460. $I_{p b}$ ranges from $810^{10}$ to $5.510^{10} p p b$ during the bunch train. Note that the $I_{p b}$ of the second group of less intense bunches (from bunch \# 17 to 33 ) causes the $E C$ signal to decrease. That may indicate a RHIC EC threshold of $I_{p b}=610^{10}$ protons per bunch $(p p b)$ for the long straight sections.

Table 1: Machine and beam specifications used for the RHIC simulations based on fill \#3460.

\begin{tabular}{cr}
\hline \hline$I_{p b}$ & $810^{10} p p b$ \\
full bunch length & $15 \mathrm{~ns}$ \\
bunch spacing & $106.6 \mathrm{~ns}$ \\
\# bunches $\left(N_{b}\right)$ & 110 \\
revolution period $\left(T_{\text {rev }}\right)$ & $12.8 \mu \mathrm{s}$ \\
rms beam radius & $2.4 \mathrm{~mm}$ \\
relativistic $\gamma$ & 25.8 \\
beam pipe radius & $6 \mathrm{~cm}$ \\
\hline \hline
\end{tabular}

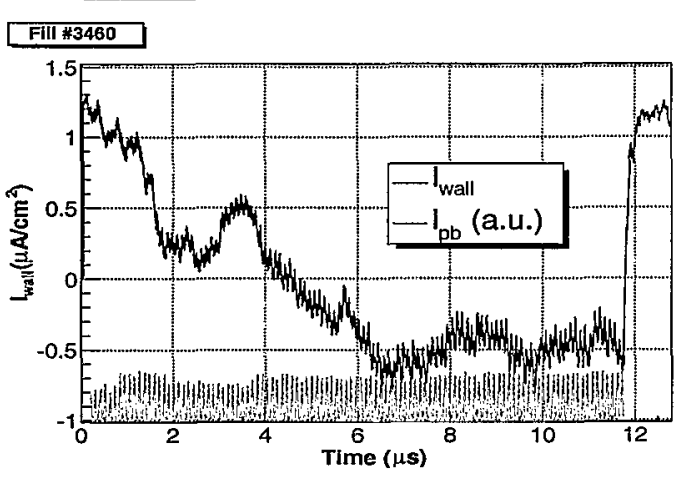

Figure 2: $E C$ signal (black solid trace) and $I_{p b}$ (red bars) for a RHIC revolution (12.8 $\mu$ s during fill 3460 ). The signal goes from positive to negative values due to the electronic design of the $E D$.

In general, the computer code simulations assume equal intensity along the bunch train. Fill $\# 3460$ can be reproduced with the recently developed $C S E C$, which allows $I_{p b}$ to be changed for different bunches. We tried to fix the wall surface parameters contributing to $E C$ by sweeping $S E Y_{\max }$ and $\mathrm{R}$ and comparing $I_{\text {wall }}$ behaviour with the experimental data. Due to the few $E C$ cases found until now, the electron dose received can be neglected. It turned out that $\mathrm{R}<0.5$ needs $S E Y_{\max }>2.2$ to multipact (far from the literature values for baked stainless steel surfaces, see [5] and [6]), whereas $R=0.7$ does not reproduce the observed decay time. Therefore, possible values for $\mathrm{R}$ are $0.6 \pm 0.1$. Figure 3 shows the $S E Y_{\max }$ sweep fixing $\mathrm{R}=0.6$. In order to determine the proper values of $S E Y_{\text {max }}$ and $\mathrm{R}$, we could fit the growth and decay times from both simulations and experimental data and compare the results. However, the experimental data comes from an $\mathrm{AC}$ coupled signal with a low frequency cut off, flow $=300 \mathrm{kHz}$ [4]. Therefore, the signal needs to be treated before fitting when slow dynamics $(<300 \mathrm{kHz})$ play a role, as they did for fill \# 3460. This treatment is being developed, and the results of the first analysis indicate that $\mathrm{R}=0.6$ and $S E Y_{\text {max }}=[1.8$ $2.0]$ match reasonably well with the estimated $I_{\text {wall }}(\approx 0.5$ $5 \frac{\mu A}{\mathrm{~cm}^{2}}$ ) and $E C$ timing (saturation $\approx 6 \mu \mathrm{s}$ ). This combination is also found in scientific papers ( [5] and [6]). The combination $\mathrm{R}=0.5$ and $S E Y_{\max } \geq 2.1$ is a possibility for the unbaked stainless steel vacuum chambers at RHIC. Note that these values are given for $R_{i n f}=0.2$. Possible consequences of changes in this parameter are currently under study.

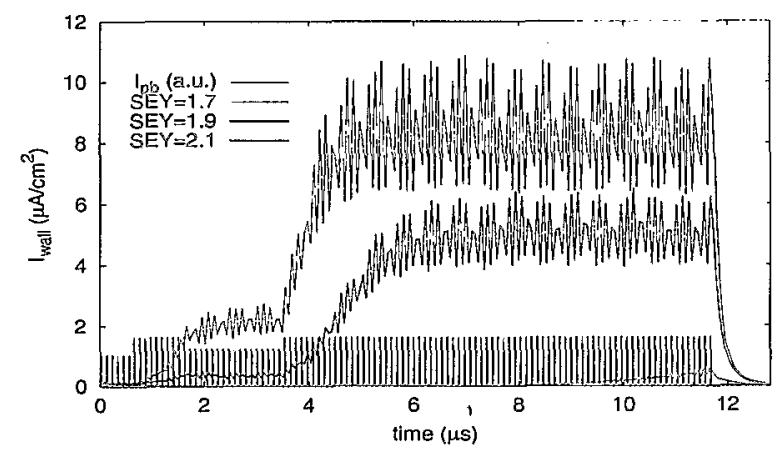

Figure 3: Sweep of possible values of $S E Y_{\max }$ fixing $\mathrm{R}=0.6$. The maximum value of the line density for these cases ranges from $0.1 \mathrm{nC} / \mathrm{m}\left(S E Y_{\max }=1.7\right)$ to $1 \mathrm{nC} / \mathrm{m}$ $\left(S E Y_{\max }=2.1\right)$.

Preliminary results using ECLOUD with the latest parametrization for elastically reflected electrons using $\delta_{\max }=1.9$ produce a line density $\rho$ at saturation on the order of $0.3 n C / m$, which is in good agreement with $C S E C$ results for $R=0.6$ and $S E Y_{\max }=1.8$.

\section{RESULTS OF APPLYING A SOLENOID FIELD}

Figure 2 shows that less intense bunches produce a strong decrease in the $E C E$ signal. Therefore, it was thought that a fill pattern with some missing gaps could avoid the $E C$ build-up. To check this possibility, RHIC was filled with the 110 bunch fill pattern, but using some gaps in the bunch train, i.e. the same beam and machine parameters stated in Table 1 except for $N_{b}=41$ and $I_{p b}=1.1$ 
$10^{11}$. In this case, 16 'filled' bunches were followed by a gap of 425ns (corresponding to 4 missing bunches). During the third bunch train, only 9 bunches were injected because of the high $P$ rise. So, the total number of bunches is $N_{b}=16+16+9=41$. Fig. 4 shows this pattern as well as the $E C$ signal. The $E C$ signal was quite evident and stable and a solenoid field $(B)$ was applied. As can be seen in Fig. 4 (top), a very weak $B$ (5.4 Gauss) already decreases the $E C$ at RHIC by a factor of $\approx 4$, and unfortunately sends the $E C$ signal below the noise level. Observations with higher $B$ did not show significant changes. Results from $C S E C$ simulations are shown on Fig.4 (bottom). According to Sec. 3, a good and reliable combination is $S E Y_{\max }=1.8$ and $R=0.6$. Simulation results for this case can be seen at Fig. 4 and confirm that $B=5.4$ Gauss is already a field strong enough to put the $E C$ signal under the RHC electron detector noise level. Further studies are currently carrying on to exactly determine the magnetic field supressing $E C$ at $\mathrm{RHIC}$.

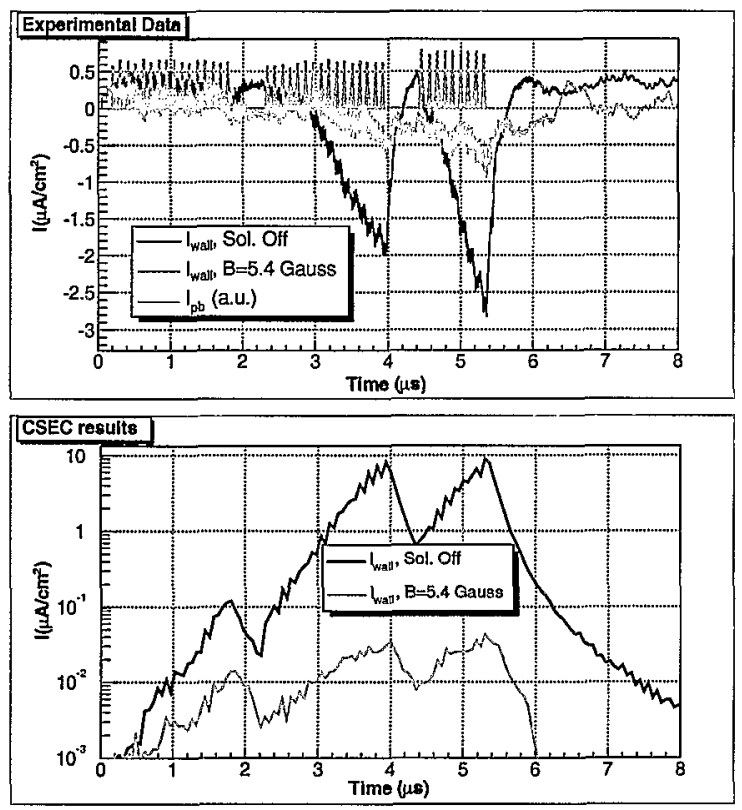

Figure 4: Experimental suppression of the $E C$ using a solenoid (top). $\mathrm{B}=5.4$ Gauss sends the $E C$ signal to the noise level. The simulation results (bottom) show good agreement. Note a logarithmic scale is used for the simulation results to highlight the difference in the order of magnitude.

\section{EVALUATION OF $P$}

For $I_{p b}$ and $N_{b}$ fixed, the final $I_{w a l l}$ is stabilized under certain conditions due to space charge effects. Observations show that the final $P$ also reaches a certain equilibrium between the desorbed gas molecules in the wall and the gas pumped by the ion pumps. This $P$ can be estimated directly proportional to $I_{\text {wall }}$ ( [1]) and depending on the the electron desorption coefficient $\left(\eta_{e}\right)$ of the beam pipe wall. This becomes a critical parameter for this calculation. Although $\eta_{e}$ changes significantly depending on the gas species, temperature, surface material and the impinging electron $E$ ( [9]), we plan to use RHIC to give a global (regardless the gas species) estimate for $\eta_{e}$ based on the experimental observations for both $I_{\text {wall }}$ and $P$. Unfortunately, the amount of data is still not enough to give a reliable value for $\eta_{e}$ and further studies are currently being carried out.

\section{CONCLUSIONS}

Observations at RHIC and simulation development are currently being carried and therefore the analysis is not definitive. However, preliminary results from simulations show good agreement with the latest experimental data $\left(I_{\text {wall }}\right.$ on the order of $\left.0.5-10 \frac{\mu A}{\mathrm{~cm}^{2}}\right)$. Both $C S E C$, $E C L O U D$ and experimental results show a $\rho \approx 0.5 \mathrm{nC} / \mathrm{m}$. We determined a value for $\mathrm{R}=0.6 \pm 0.1$ for $S E Y_{\max }$ (1.8-2.1) for baked stainless steel vacuum chambers used; and $S E Y_{\max }>2.1$ for the unbaked case using $C S E C$ parametrization. However, variations on $R_{\text {inf }}$ have not been considered, and these values may vary slightly. Weak solenoid fields $(\approx 10$ Gauss) are strong enough to suppress the cloud for $I_{p b} \approx 10^{11} \mathrm{ppb}$. We introduced also a preliminary plan to find a global value for $\eta_{e}$ from the observations of $P$ and $I_{\text {wall }}$.

\section{ACKNOWLEDGEMENTS}

The authors would like to thank D. Gassner, J. Gullotta, O. Gould, P. He, H.C. Hseuh, R. Lee, V. Ponnayiyan, L. Smart, D. Trbojevic and S.Y. Zhang for their precious help in the data acquisition and understanding. The use of $E C L O U D$ would have not been possible without $\mathrm{G}$. Rumolo.

\section{REFERENCES}

[1] W.Fischer et al. Vacuum pressure rise with intense ion beams in RHIC. EPAC'02, Paris, 2002.

[2] M. Blaskiewicz et al. Electron cloud instabilities in the PSR and SNS. PRST-AB 6, 014203. Jan. 2003.

[3] G. Rumolo and F. Zimmermann. Practical User Guide for ECloud. SL-Note-2002-016 AP. CERN, Geneva, May 2002.

[4] U. Iriso-Ariz et al. Electron detectors for vacuum pressure rise diagnostics at RHIC. In these proceedings.

[5] M. A. Furman and M. Pivi. Probabilistic model for the simulation of secondary electron emission. PRST-AB 5, 124404, Dec. 2002.

[6] C. Scheuerlein et al. The SEY of technical materials and its variation with surface treatments. Proceedings of EPAC'00, Vienna, June 2000.

[7] F. Zimmermann. Electron Cloud Progress, 13th LHC MAC. March 2003.

[8] S.Y. Zhang et al. RHIC pressure rise and electron cloud. In these proceedings.

[9] J. Gomez-Goni and A.G. Mathewson. Temperature dependence of the electron induced gas desorption yields on stainless steel, copper and aluminum. J. Vac. Sci. Technol. A 15 (6), Nov/Dec 1997. 